COMPUTATIONAL METHODS IN APPLIED MATHEMATICS, Vol.5(2005), No.2, pp.155-169

(C) 2005 Institute of Mathematics of the National Academy of Sciences of Belarus

\title{
GENERAL KINETIC MODELS FOR VEHICULAR TRAFFIC FLOWS AND MONTE-CARLO METHODS
}

\author{
M. HERTY, A. KLAR \\ TU Kaiserslautern, Fachbereich Mathematik \\ Postfach 3049, D-67653 Kaiserslautern, Germany \\ L. PARESCHI \\ University of Ferrara, Department of Mathematics \\ Via Machiavelli 35, I-44100 Ferrara, Italy
}

\begin{abstract}
In this paper we present a general derivation of kinetic models for traffic flows including different kinds of interaction rules. We show that most kinetic previously derived models can be cast in the actual formulation. The development of Monte-Carlo methods for direct simulation of kinetic models is considered as an initial step towards realistic and efficient computations of traffic phenomena. MonteCarlo methods are developed for these kinetic models. Several numerical examples are computed and compared to the previously obtained solutions of the stationary equation.
\end{abstract}

2000 Mathematics Subject Classification: 82C40; 65C05; 82C 80 .

Keywords: kinetic equations, stationary solutions, Monte-Carlo methods.

\section{Introduction}

We present a general framework for kinetic traffic flow models and a new Monte-Carlo method for computing stationary solutions. The kinetic or Boltzmann-like traffic flow models have been the subject of several discussions by different authors, e.g., [2,4-8, 12-14]. We do not intend to restate the complete discussion. Nowadays kinetic models are accepted as an intermediate step between macroscopic and microscopic models [6].

The Monte-Carlo methods are widely used to simulate the dynamics of complex systems. They have many advantages, of which the simplicity and reduced computational cost make them very attractive for realistic simulations. In the kinetic theory of rarefied gases, for example, they have been widely used to simulate stationary flows (see $[1,9,10]$ for a recent review on such methods). In traffic flows, however, such methods have not been used, at least in connection with partial differential equations. We refer to [15] for direct stochastic simulations starting from microscopic probabilistic considerations.

In this paper we start from the general microscopic interaction rules to derive the corresponding kinetic equations. Furthermore, we introduce a numerical scheme based on the 
Monte-Carlo methods to compute the stationary solution in the homogeneous case. The derivation of kinetic equations by considering single driver interactions was also done in $[6,7]$ for special interaction rules. We present here a more general approach including also more sophisticated driver dynamics as presented for example in [3]. Numerical results for the different stationary solution are given. The latter are obtained by applying a suitable stochastic particle method to the traffic flow models. More details on the numerics can also be found in $[10,11]$. This homogenous study represents a preliminary step towards the use of MonteCarlo methods in the simulation of traffic flows problems.

\section{Basic equations}

Below we consider only the single lane models. The concepts can easily be extended to multilane models. By $f(x, t, v)$ we denote the density of cars that at time $t$ are at location $x$ and are driving with velocity $v$. Here, $x \in \mathbb{R}$ denotes the location on the highway; we assume $v \in\left[0, v_{\max }\right]$, where $v_{\max }$ is the maximal allowed speed. We can normalize $v_{\max }$ to $v_{\max }=1$.

We define the following macroscopic quantities: $\rho(x, t)=\int_{0}^{1} f(x, t, v) d v$ is the car density at location $x$ and time $t$. The density may vary in $\left[0, \rho_{\max }\right]$ where we normalize $\rho_{\max }=1$. The macroscopic flux is given by $j(t, x)=\int_{0}^{1} v f(x, t, v) d v$.

The general kinetic traffic flow model is given by the following evolution equation for the distribution function $f=f(x, t, v)$

$$
\partial_{t} f+v \partial_{x} f=G\left(f, f^{2}, x, x+H_{G}, t, v\right)-L\left(f, f^{2}, x, x+H_{L}, t, v\right) .
$$

In this notation the change in the distribution function $f$ is given by gain terms denoted by $G$ and loss terms $(L)$. Usually, gain and loss are due to acceleration and braking scenarios, respectively. The vehicular traffic flow models under consideration deal with the interaction of only two cars. Hence, we obtain a dependence of the source term on $f^{2}\left(x, t, H, v, v^{\prime}\right)$. This term describes the distribution function of a pair. The source term may also include non-local terms $x+H_{L, G}$. These terms are due to drivers who act according to the car in front of them (having a positive distance $H_{L}, H_{G}$ )

To simplify the equation, we assume the following approximation

$$
f^{2} \approx q f(x, v) f\left(x+H, v^{\prime}\right)
$$

with a known function $q$. This is similar to [6]. We consider the space-homogenous situation for various driver interactions. The different possible interaction rules are taken from vehicular flow models like those given in $[2-4,6]$.

\subsection{Driver interactions}

The general kinetic equation (1) can be derived by describing the microscopic interaction rules. Common to all models are the following three different behaviors of vehicular car drivers.

We consider two cars with velocities $v$ and $w$. The possible interactions are acceleration, braking and reaching a desired velocity. They are given as follows.

1. Acceleration. If $v<w$, then the car with velocity $v$ will accelerate to $v^{+}$according to the following law:

$$
v^{+}=v+a(v, w, \xi) .
$$


The function a may also depend on a random variable $\xi$. This takes into account the fact that the acceleration process is not deterministic, but depends on the actual driver.

2. Braking. If $v>w$, then the car with velocity $v$ will brake to prevent an accident and reach a new velocity $v^{\prime}$ :

$$
v^{+}=v-b(v, w, \xi)
$$

3. Independent of the velocity of other cars, a driver may want to reach his desired velocity. This can be modelled by introducing an additional interaction rule like

$$
v^{+}=d(v, \xi)
$$

The models of Klar et al. and Helbing are included in the above framework. We give some examples. Let $\xi$ denote a stochastic variable uniformly distributed in $[0,1]$.

Example 1. Model of Illner, Klar et al. [4].

$$
a(v, w, \xi)=(1-\xi)\left(v_{\max }-v\right),
$$

where $v_{\max }$ is the maximal allowed velocity of the cars.

$$
\begin{aligned}
b(v, w, \xi) & =\xi v \\
d(v, \xi) & =0 .
\end{aligned}
$$

Example 2. Simplified model of Klar et al. [2].

$$
\begin{aligned}
a(v, w, \xi) & =(1-\xi)(w-v), \\
b(v, w, \xi) & =\xi(v-w), \\
d(v, \xi) & =\xi v_{\max } .
\end{aligned}
$$

Example 3. Helbing-like [3] interaction rule. Following [3], the complete microscopic dynamics for a single car $\alpha$ at time $t$ with position $x_{\alpha}(t)$ and velocity $v_{\alpha}(t)$ are

$$
\begin{aligned}
x_{\alpha}^{\prime}(t) & =v_{\alpha}(t), \\
v_{\alpha}^{\prime}(t) & =\tilde{a}\left(1-\left(\frac{v_{\alpha}}{v_{0}}\right)^{\delta}-\left(\frac{s_{*}}{s}\right)^{2}\right), \\
s_{*} & =s_{0}+\max \left(v_{\alpha} T+\frac{v_{\alpha}\left(v_{\alpha}-v_{\alpha-1}\right)}{2 \sqrt{\tilde{a} \tilde{b}}}, 0\right) .
\end{aligned}
$$

Here, $s=\left[x_{\alpha-1}(t)-x_{\alpha}(t)-l_{\alpha}\right]$ is the gap between the vehicle $\alpha$ and the car $\alpha-1$, moving in front. Further, $\tilde{a}, \delta$ and $\tilde{b}$ are the acceleration and braking parameters and $v_{\alpha} T$ is the safety distance, $s_{0}$ is the minimum distance to a standing vehicle.

We rewrite the different acceleration and braking factors in terms of $a, b$ and $d$ given by $(3,4)$ and $(5)$, respectively. These equations can be seen as simple discretizations of $(12)$.

Note that model (12) is deterministic and, therefore, there is no dependence on the stochastic variable. 
The acceleration on a clear road is given by $\tilde{a}\left(1-\left(v / v_{0}\right)^{\delta}\right)$, where $v_{0}$ is the desired velocity. The exponent $\delta$ is in the range of $[1, \ldots, 5]$ depending on the acceleration behavior of the drivers. Hence,

$$
d(v)=v+\tilde{a}\left(1-\left(v / v_{0}\right)^{\delta}\right) .
$$

The braking term is given by $-\tilde{a}\left(s^{*} / s_{\alpha}\right)^{2}$. Since we consider a spatially homogeneous situation, we obtain $s_{\alpha}=l_{\alpha}=$ const and

$$
b(v, w)=\tilde{a}\left(s^{*} / s_{\alpha}\right)^{2}, s^{*}=\left(s_{0}+v T\right)+v(v-w) / \sqrt{2 \tilde{a} \tilde{b}} .
$$

Except for the term $d$, there is no additional acceleration term

$$
a(v, w, \xi)=0 .
$$

For further explanation on the choice of the constants $\tilde{a}$ and $\tilde{b}$, refer to [3].

Several other kinetic traffic flow models can be found, for example, in $[2,6]$ and the references therein.

\subsection{Derivation of kinetic equations}

Combining the driver interactions with (1), we obtain a general traffic model. We will give some examples for the final equations.

From the above driver interaction rules we can derive the general form (1) with the corresponding gain and loss terms. The evolution equation in the space-homogeneous case is given by

$$
\partial_{t} f=G(f, t, v)-L(f, t, v) .
$$

The subscript $A, B$ denotes the type of interaction corresponding to the gain or loss at the point $(t, v)$. We have

$$
G(f, v)=G_{A}(f, v)+G_{B}(f, v)+G_{D}(f, v),
$$

where $N^{A, B, D}$ are sets of points $\xi$ depending on $v$ and $w$ :

$$
\begin{aligned}
G_{A}(f, v) & =\int_{0}^{v_{\max }} \int_{N^{A}(v, w)}|\operatorname{det} J| \beta_{\left(v^{\prime}, w\right) \mapsto(v, w)}^{A} f\left(v^{\prime}\right) f(w) d \xi d w, \\
N^{A}(v, w) & :=\left\{\xi: 0 \leqslant \xi \leqslant 1, v^{\prime}<w, 0 \leqslant v^{\prime} \leqslant v_{\max }, v=v^{\prime}+a\left(v^{\prime}, w, \xi\right)\right\}, \\
G_{B}(f, v) & =\int_{0}^{v_{\max }} \int_{N^{B}(v, w)}|\operatorname{det} J| \beta_{\left(v^{\prime}, w\right) \mapsto(v, w)}^{B} f\left(v^{\prime}\right) f(w) d \xi d w, \\
N^{B}(v, w) & :=\left\{\xi: 0 \leqslant \xi \leqslant 1, v^{\prime}>w, 0 \leqslant v^{\prime} \leqslant v_{\max }, v=v^{\prime}-b\left(v^{\prime}, w, \xi\right)\right\}, \\
G_{D}(f, v) & =\int_{N^{D}(v)}|\operatorname{det} J| \beta_{v^{\prime} \mapsto v}^{D} f\left(v^{\prime}\right) d \xi, \\
N^{D}(v) & :=\left\{\xi: 0 \leqslant \xi \leqslant 1,0 \leqslant v^{\prime} \leqslant v_{\max }, v=d\left(v^{\prime}, \xi\right)\right\} .
\end{aligned}
$$


Here, $J$ is the Jacobian of the corresponding transformation $(v, w) \mapsto\left(v^{\prime}, w\right)$ s.t. $v=$ $v^{\prime}+a\left(\xi, v^{\prime}, w\right), v=v^{\prime}-b\left(\xi, v^{\prime}, w\right)$ and $v=d\left(v^{\prime}, \xi\right)$ respectively. Note that in all the above formulas $v^{\prime}$ is given implicitly and $v, w$ and $\xi$ are the parameters. For given functions $a, b$ and $d$ the above formulas are simplified and the terms can be expressed explicitly in terms of $v^{\prime}$ and $w$. We refer to the examples in the next subsection for further details.

The loss terms $L(f, t, v)=L_{A}+L_{B}+L_{D}$ are given by

$$
\begin{aligned}
& L_{A}(f, v)=\int_{v<w} \beta_{(v, w) \mapsto\left(v^{\prime}, w\right)}^{A} f(v) f(w) d w, \\
& L_{B}(f, v)=\int_{v>w} \beta_{(v, w) \mapsto\left(v^{\prime}, w\right)}^{B} f(v) f(w) d w, \\
& L_{D}(f, v)=f(v) \int \beta_{v \mapsto v^{\prime}}^{D} d v^{\prime}=f(v) .
\end{aligned}
$$

The functions $\beta^{A}, \beta^{B}$ and $\beta^{D}$ consist of the correlation function $q$ introduced above and further modelling parameters describing the strength of the microscopic interaction.

We have the following remark on the inhomgenous situation. Inhomogeneous traffic equations can be derived in the usual way, see, for example, the publications cited in the introduction, especially $[5,6]$.

\subsection{Examples}

We derive the Illner-Klar model, the simplified Klar model and the Helbing model from the above microscopic setting. We give the numerical results on these three models in Section 4 . We assume single lane models and the maximum velocity $v_{\max }=1$.

2.3.1. Example 1: the model of Illner, Klar et al. The acceleration and braking behavior is given by

$$
a(v, w, \xi)=(1-\xi)(1-v), \quad b(v, w, \xi)=\xi v,
$$

where $0 \leqslant \xi \leqslant 1$. Further,

$$
\beta^{A}\left(v^{\prime}, w\right)=\left|v^{\prime}-w\right| q_{A}(\rho) / \rho, \quad \beta^{B}\left(v^{\prime}, w\right)=\left|v^{\prime}-w\right| q_{B}(\rho) / \rho,
$$

where $q_{A}$ and $q_{B}$ are correlation functions depending on the macroscopic quantities only.

Consider the case of acceleration first. The transformation $F=\left(F_{1}, F_{2}\right):(v, w) \mapsto\left(v^{\prime}, w\right)$ is given by $v=v^{\prime}+a\left(v^{\prime}, w, \xi\right)$ and $w=w$, i.e.,

$$
F(v, w ; \xi)=\left[\begin{array}{c}
\frac{v-1+\xi}{\xi} \\
w
\end{array}\right]
$$

Further, $J=\nabla F$ with $|\operatorname{det} J|=\frac{1}{\xi}$ and $\partial_{\xi} F_{1}(v, w ; \xi)=\left(1-v^{\prime}\right)|\operatorname{det} J|$. Using $v=v^{\prime}+$ $a\left(v^{\prime}, w, \xi\right)$, we have

$$
G_{A}(f, v)=\int_{0}^{1} \int_{N(v, w)}|\operatorname{det} J| \beta^{A}\left(v^{\prime}, w\right) f\left(v^{\prime}\right) f(w) d \xi d w
$$


where the set $N(v, w)$ is given by $N(v, w)=\left\{\xi: 0 \leqslant \xi \leqslant 1, v^{\prime}<w, 0 \leqslant v^{\prime} \leqslant 1, v=v^{\prime}+\right.$ $\left.a\left(v^{\prime}, w, \xi\right)\right\}$. Next, we transform the coordinates, i.e., we apply the transformation formula to $G_{A}$ with $\xi \mapsto v^{\prime}$ and $v^{\prime}=F_{1}(v, w, \xi)$. Let $M(v, w)=\left\{v^{\prime}: 0 \leqslant v^{\prime} \leqslant 1, v^{\prime}<w, 0 \leqslant \frac{v-v^{\prime}}{1-v^{\prime}} \leqslant 1\right\}$, then we obtain

$$
G_{A}(f, v)=\int_{0}^{1} \int_{M(v, w)} \beta^{A}\left(v^{\prime}, w\right) \frac{1}{1-v^{\prime}} f\left(v^{\prime}\right) f(w) d v^{\prime} d w
$$

Reformulating the conditions describing the set $M(v, w)$ and inserting $\beta^{A}$, we get

$$
G_{A}(f, v)=\frac{1}{\rho} \int_{0}^{1} \int_{v^{\prime}<w, 0 \leqslant v^{\prime} \leqslant 1} q_{A}(\rho)\left|v^{\prime}-w\right| f\left(v^{\prime}\right) f(w) \frac{1}{1-v^{\prime}} \chi_{\left[v^{\prime}, 1\right]}(v) d v^{\prime} d w .
$$

This is the considered gain term due to the acceleration as in [4].

Next, we consider the gain term due to the braking. The transformation $F$ is given by

$$
F(v, w, \xi)=\left[\begin{array}{c}
v /(1-\xi) \\
w
\end{array}\right]
$$

and $|\operatorname{det} J|=1 /(1-\xi)$ and $\partial_{\xi} F_{1}(v, w, \xi)=|\operatorname{det} J| v^{\prime}$. Define $N(v, w):=\left\{\xi: 0 \leqslant v^{\prime} \leqslant 1,0 \leqslant\right.$ $\left.\xi \leqslant 1, v^{\prime}>w, v=v^{\prime}-b\left(v^{\prime}, w, \xi\right)\right\}$, then for $v^{\prime}=v /(1-\xi)$

$$
G_{B}(f, v)=\int_{0}^{1} \int_{N(v, w)} \partial_{\xi} F_{1}(v, w ; \xi) /\left(1-v^{\prime}\right) \beta^{B}\left(v^{\prime}, w\right) f\left(v^{\prime}\right) f(w) d \xi d w .
$$

Let $M(v, w)=\left\{v^{\prime}: 0 \leqslant v^{\prime} \leqslant 1,0 \leqslant v / v^{\prime} \leqslant 1, v^{\prime}>w\right\}=\left\{v^{\prime}: 0 \leqslant v^{\prime} \leqslant 1,0 \leqslant v \leqslant v^{\prime}, v^{\prime}>w\right\}$ and apply the transformation formula for $\xi \mapsto v^{\prime}$ to obtain

$$
\begin{aligned}
G_{B}(f, v) & =\int_{0}^{1} \int_{M(v, w)} \frac{1}{v^{\prime}} \beta^{B}\left(v^{\prime}, w\right) f\left(v^{\prime}\right) f(w) d v^{\prime} d w \\
& =\int_{0}^{1} \int_{v^{\prime}>w, 0 \leqslant v^{\prime} \leqslant 1} \frac{1}{v^{\prime}} \chi_{\left[0, v^{\prime}\right]}(v) \beta^{B}\left(v^{\prime}, w\right) f\left(v^{\prime}\right) f(w) d v^{\prime} d w .
\end{aligned}
$$

After inserting the definition of $\beta^{B}$ we obtain the gain term due to the braking as in [4].

The derivation of the loss terms is easy and we skip the details.

Assume $\forall \rho: q^{A}(\rho) \neq 0$. We introduce

$$
k(\rho):=\frac{q^{B}(\rho)}{q^{A}(\rho)}, \quad c(\rho)=q^{A}(\rho) / \rho
$$


and summarize the above considerations:

$$
\begin{aligned}
f_{t}(v, t)= & c(\rho)\left(k(\rho) \int_{0}^{1} \int_{v^{\prime}>w}\left|v^{\prime}-w\right| f\left(v^{\prime}, t\right) f(w, t) \frac{1}{v^{\prime}} \chi_{\left[0, v^{\prime}\right]}(v) d v^{\prime} d w\right. \\
& +\int_{0}^{1} \int_{v^{\prime}<w}\left|v^{\prime}-w\right| f\left(v^{\prime}, t\right) f(w, t) \frac{1}{1-v^{\prime}} \chi_{\left[v^{\prime}, 1\right]}(v) d v^{\prime} d w \\
& -k(\rho) \int_{v>w}|w-v| f(w, t) f(v, t) d w \\
& \left.-\int_{v<w}|w-v| f(w, t) f(v, t) d w\right) \\
= & : c\left(k \tilde{G}_{B}+\tilde{G}_{A}-k \tilde{L}_{B}-\tilde{L}_{A}\right) .
\end{aligned}
$$

Note that in equation (18) the operators $\tilde{G}_{A, B}$ and $\tilde{L}_{A, B}$ have the same kernel $\tilde{\beta}(v, w)=$ $|v-w|$.

\subsubsection{Example 2: the simplified model of Klar et al. We have}

$$
a(v, w, \xi)=(1-\xi)(w-v), \quad b(v, w, \xi)=\xi(v-w)
$$

and again

$$
\beta^{A}\left(v^{\prime}, w\right)=\left|v^{\prime}-w\right| q_{A}(\rho) / \rho, \quad \beta^{B}\left(v^{\prime}, w\right)=\left|v^{\prime}-w\right| q_{B}(\rho) / \rho, \quad \beta^{D}=1 .
$$

Consider the gain term due to the acceleration. As before, we derive the transformation $F$ from $v=v^{\prime}+a(v, w, \xi)$ :

$$
F(v, w, \xi)=\left[\begin{array}{c}
\frac{v-w(1-\xi)}{\xi} \\
w
\end{array}\right]
$$

and $|\operatorname{det} J|=1 / \xi$ and $\partial_{\xi} F_{1}(v, w, \xi)=\left(w-v^{\prime}\right) \operatorname{det} J$. Then a similar calculation shows

$$
\begin{aligned}
G_{A}(f, v) & =\int_{0}^{1} \int_{N(v, w)}|\operatorname{det} J| \beta^{A}\left(v^{\prime}, w\right) f\left(v^{\prime}\right) f(w) d \xi d w \\
& =\frac{1}{\rho} \int_{0}^{1} \int_{0 \leqslant v^{\prime} \leqslant 1, v^{\prime}<w}\left|v^{\prime}-w\right| q^{A}(\rho) f\left(v^{\prime}\right) f(w) \frac{1}{w-v^{\prime}} \chi_{\left[v^{\prime}, w\right]}(v) d v^{\prime} d w,
\end{aligned}
$$

where $N(v, w)=\left\{\xi: 0 \leqslant \xi \leqslant 1, v^{\prime}<w, 0 \leqslant v^{\prime} \leqslant 1, v=v^{\prime}+a\left(v^{\prime}, w, \xi\right)\right\}$. Consider the gain due to the braking. It is sufficient to notice that

$$
F(v, w, \xi)=\left[\begin{array}{c}
\frac{v-w}{1-\xi}+w \\
w
\end{array}\right]
$$

and that $\operatorname{det} J=1 /(1-\xi)$. Then we follow a similar procedure as in the previous example. Finally, we consider the gain term due to the free acceleration. Since the maximal velocity is equal to one, we obtain

$$
d(v, \xi)=\xi=v^{\prime}
$$


and $v^{+}=d(v, \xi)$ as a microscopic interaction law. To compute the gain term $G_{D}(f, v)$, we consider the transformation $F$ given by $v^{\prime}=d(v, \xi)$

$$
F(v, \xi)=[\xi] \text {. }
$$

In this case $|\operatorname{det} J|=1$ and we obtain

$$
G_{D}(f, v)=\int_{\left\{\xi: 0 \leqslant \xi \leqslant 1, v=\xi=v^{\prime}\right\}}|\operatorname{det} J| \beta^{D}\left(v^{\prime}\right) f\left(v^{\prime}\right) d \xi=\int_{0}^{1} f\left(v^{\prime}\right) d v^{\prime} .
$$

This coincides with the gain term due to the free acceleration in [2]. The equivalence of the loss terms follows immediately. We omit the details.

Using the notation $k(\rho)=q^{B}(\rho) / q^{A}(\rho), c(\rho)=q^{A}(\rho) / \rho$, we obtain the full equation:

$$
\begin{aligned}
f_{t}(v, t)= & c(\rho)\left(k(\rho) \int_{0}^{1} \int_{v^{\prime}>w}\left|v^{\prime}-w\right| f\left(v^{\prime}, t\right) f(w, t) \frac{1}{v^{\prime}-w} \chi_{\left[w, v^{\prime}\right]}(v) d v^{\prime} d w\right. \\
& +\int_{0}^{1} \int_{v^{\prime}<w}\left|v^{\prime}-w\right| f\left(v^{\prime}, t\right) f(w, t) \frac{1}{w-v^{\prime}} \chi_{\left[v^{\prime}, w\right]}(v) d v^{\prime} d w \\
& -k(\rho) \int_{v>w}|w-v| f(w, t) f(v, t) d w \\
& \left.-\int_{v<w}|w-v| f(w, t) f(v, t) d w\right) \\
= & : c\left(k \tilde{G}_{B}+\tilde{G}_{A}-k \tilde{L}_{B}-\tilde{L}_{A}+1 / c \tilde{G}_{S}-1 / c \tilde{L}_{S}\right) .
\end{aligned}
$$

Again, the kernels of $\tilde{G}_{A, B}$ and $\tilde{L}_{A, B}$ coincide.

2.3.3. Example 3: The Helbing-like model In these models the acceleration term $a=0$ and $b, d$ do not depend on the random variable $\xi$. Further, in all cases the macroscopic strength of the interaction is equal to one, i.e., $\beta^{B}=\beta^{D}=1$.

We obtain for the gain term due to the free acceleration $G_{D}(f, v)$ the transformation $F(v)=v^{\prime}$. Here $v^{\prime}$ is given implicitly by $v=v^{\prime}+\tilde{a}\left(1-\left(v^{\prime} / v_{0}\right)^{\delta}\right)$. Further,

$$
G_{D}(f, v)=|\operatorname{det} J| f\left(v^{\prime}\right) \int_{0}^{1} 1 d \xi
$$

Now consider the braking term given by (14). We obtain $G_{B}(f, v)$ as

$$
G_{B}(f, v)=\int_{v^{\prime}>w}|\operatorname{det} J| f\left(v^{\prime}\right) f(w) d w \int_{0}^{1} 1 d \xi,
$$

where $F(v, w)=\left(v^{\prime}, w\right)$ and $v^{\prime}$ is defined implicity by the formula

$$
v=v^{\prime}-\tilde{a}\left(s^{*} / s_{\alpha}\right)^{2} \text { and } s^{*}:=\left(s_{0}+v^{\prime} T\right)+v^{\prime}\left(v^{\prime}-w\right) / \sqrt{2 \tilde{a} \tilde{b}} .
$$


Again the derivation of the loss terms is similar and omitted. Finally,

$$
f_{t}(v, t)=\tilde{G}_{D}-\tilde{L}_{D}+\tilde{G}_{B}-\tilde{L}_{B}
$$

Starting from the general form (18) (which includes (18) and (19)), we derive a MonteCarlo algorithm to compute stationary solutions in the next section.

\section{Monte-Carlo algorithm for stationary solutions}

In order to simplify the description of the method and the notations we make the following assumptions on $\beta^{A}$ and $\beta^{B}$

$$
\beta^{A}(v, w)=\left\{\begin{array}{ll}
\beta(v, w), & v<w, \\
0, & \text { otherwise }
\end{array} \quad \beta^{B}(v, w)= \begin{cases}k \beta(v, w), & w<v \\
0, & \text { otherwise }\end{cases}\right.
$$

where $k$ is a function depending only on macroscopic quantities. The factor $k$ is a weighting parameter that can control the relation between the acceleration and braking forces. The assumption is satisfied by all models of Klar et al. [6] and also by the Illner, Klar model [4].

We start with the general time-dependent equation

$$
f_{t}=k G_{B}+G_{A}-k L_{B}-L_{A}+G_{D}-L_{D}
$$

where now the value $k$ has been emphasized so that $G_{A, B}$ and $L_{A, B}$ are defined through the same kernel $\beta$ and all integrations are performed on the whole velocity range.

Note that in (21) the loss operator due to the free flow is given by

$$
L_{D}=f(v) .
$$

First, we split the process into acceleration and braking due to the car-car interaction and single car interactions

$$
\begin{aligned}
& f_{t}=k G_{B}+G_{A}-k L_{B}-L_{A}, \\
& f_{t}=G_{D}-L_{D} .
\end{aligned}
$$

In order to develop a Monte-Carlo algorithm, we replace the gain operators by

$$
\begin{aligned}
G_{A} & =\iint \beta^{A}\left(v^{\prime}, w\right) f\left(v^{\prime}\right) f(w) d\left(v^{\prime}, w\right) \\
& =\iint \min \left\{\Sigma, \beta^{A}\left(v^{\prime}, w\right)\right\} f\left(v^{\prime}\right) f(w) d\left(v^{\prime}, w\right),
\end{aligned}
$$

and similarly the loss operators by

$$
\begin{aligned}
L_{A} & =f(v) \int \beta^{A}(v, w) f(w) d w \\
& =f(v) \Sigma \rho-f(v) \int\left(\Sigma-\min \left\{\Sigma, \beta^{A}\left(v^{\prime}, w\right)\right\}\right) f(w) d w \\
& =\Sigma \rho\left(f(v)-\tilde{L_{A}}\right),
\end{aligned}
$$

where $\Sigma>0$ is a suitable constant usually referred to as dummy cross section in rarefied gas simulations. 
The same reformulations are carried out also for the braking terms $G_{B}$ and $L_{B}$. Note that at the level of particles these identities are trivial for a suitable choice of $\Sigma$. In fact, due to the finite number of car-particles we can simply define $\Sigma=\max _{i, j} \beta\left(v_{i}, v_{j}\right)$. In the continuous case, the boundedness of the cross section follows rather naturally in traffic flows thanks to the finite maximum velocity that a car can attain.

If we apply the explicit Euler method to (23) we obtain

$$
f^{n+1}=f^{n}(1-\mu \Delta t)+\mu \Delta t\left(\frac{k}{k+1}\left(\tilde{G}_{B}+\tilde{L}_{B}\right)+\frac{1}{k+1}\left(\tilde{G}_{A}+\tilde{L}_{A}\right)\right),
$$

where $\mu=\Sigma \rho(k+1)$ and $\tilde{G_{A, B}}=G_{A, B} /(\Sigma \rho)$.

The probabilistic interpretation for $\Delta t<1 / \mu$ is as follows.

- With probability $(1-\mu \Delta t)$ a car does not change its speed $v$.

- With probability $\mu \Delta t$ a car undergoes a possible change in its speed $v$.

- With probability $k /(k+1)$ a car undergoes a possible breaking scenario.

* Given a second car with velocity $w$ breaking occurs if $v>w$ with the relative probability $\beta(v, w) / \Sigma$.

- With probability $1 /(k+1)$ a car undergoes a possible acceleration scenario.

* Given a second car with velocity $w$ acceleration occurs if $v<w$ with the relative probability $\beta(v, w) / \Sigma$.

Next, if we apply the explicit Euler method to (24) we have

$$
f^{n+1}=f^{n}(1-\Delta t)+\Delta t G_{D}
$$

Thus, for $\Delta t<1$ with probability $\Delta t$ a car changes its velocity towards the desired one.

The corresponding Monte-Carlo algorithm for solving (21) is as follows

\section{ALGORITHM}

1. Sample $N$ particles from the initial distribution $f^{0}(v)$

2. Choose $\Delta t$ sufficiently small

3. Loop until stationary state is reached

(a) Treatment of the gain and loss due to car-car interactions.

Compute the number of particles $N_{1}=(1-\Delta t \mu)$ which do not interact. Select randomly $N_{c}=N-N_{1}$ cars which may interact.

Choose $N_{c} / 2$ pairs of particles described by their velocities $\left(v_{i}, v_{j}\right)$. For each pair let $\xi_{1,2,3} \in[0,1]$ be uniformly distributed random variables. Calculate $\Sigma$. Calculate $\min \left\{\Sigma, \beta\left(v_{i}, v_{j}\right)\right\}:=\Sigma_{i j}$. Update the velocities according to the following rule.

i. Possible braking scenario.

If $\xi_{1} \leqslant \frac{k}{k+1}$ and if $v_{i}>v_{j}$ and if $\xi_{2} \Sigma<\Sigma_{i j}$ update by

$$
v_{i}^{\prime}=v_{i}-b\left(v_{i}, v_{j}, \xi_{3}\right), v_{j}^{\prime}=v_{j} .
$$


ii. Possible acceleration scenario.

If $\xi_{1} \geqslant \frac{1}{k+1}$ and if $v_{i}<v_{j}$ and if $\xi_{2} \Sigma<\Sigma_{i j}$ update by

$$
v_{i}^{\prime}=v_{i}+a\left(v_{i}, v_{j}, \xi_{3}\right), v_{j}^{\prime}=v_{j} .
$$

(b) Treatment of the gain and loss due to the free flow interaction.

Compute the number of particles $N_{1}=(1-\Delta t) N$ which do not interact. Choose $N_{c}=N-N_{1}$ particles which will change their velocity. Each of the $N_{c}$ particles changes its velocity $v$ according to the rule implied by $G_{D}$, i.e.,

$$
v^{\prime}=d(v, \xi)
$$

\section{Numerical results}

We used the Monte-Carlo scheme introduced in the previous section to compute stationary solutions for different models. The computation is performed by using 20 '000 particles. The plots are generated with 200 points of reconstruction for each curve.

\subsection{Example 1}

The first example is the model of Illner, Klar et al. [4]. The driver interactions are given by

$$
a(v, w, \xi)=(1-\xi)\left(v_{\max }-v\right), \quad b(v, w, \xi)=\xi v, \quad d(v, \xi)=0
$$

where $v_{\max }$ is normalized to 1 . The kernels of the gain and loss terms are

$$
\beta^{A}=|v-w| / \rho, \quad \beta^{B}=k|v-w| / \rho, \quad \beta^{D}=0,
$$

where we assume $k>0$ to be constant. The relation between the acceleration and braking forces is expressed by $k$. We compute the stationary solutions to the model (18) for different choices of $k$. The stationary solutions are given in Fig. 1. The results coincide with the considerations in [4]. Especially, we observe that the solution is symmetric with a unique maximum. The same solutions are given in Fig. 2 in a log-log plot. We observe that for all $k$ and for $v \rightarrow 0$ the sationary solutions have a powerlaw decay.

\subsection{Example 2}

The second example is the Klar and Wegener model [2]. Again we choose $\beta^{A, B}=|v-w| / \rho$ and $k=1$. The driver interactions are given by

$$
a(v, w, \xi)=(1-\xi)(w-v), b(v, w, \xi)=\xi(w-v) .
$$

We compute the stationary solution for the case $G_{D}=0$, i.e., $d(v, \xi)=0$ and for $d(v, \xi)=$ $\xi v_{\max }$. The results are given in Fig. 3.

\subsection{Example 3}

The last example is the "intelligent driver model" proposed by Helbing et al. We set $\beta^{A, B, C}=$ 1 and $k=1$. The driver interactions are as in case 4 in Section 2.1. The parameters for the simulation are taken from [3]:

$$
\tilde{a}=0.6, \quad \tilde{b}=0.9 \text { and } T=1.5 .
$$


We assume the desired velocity of the cars to be $v_{0}=0.5$. We present stationary solutions for different values of $\delta$. As pointed out in [3], $\delta$ controls the acceleration behaviour of the drivers towards their desired velocity. It varies between the constant acceleration $(\delta \rightarrow \infty)$ and the exponential acceleration behaviour $(\delta=1)$. The effect of varying $\delta$ on the stationary solutions is given in Fig. 4.

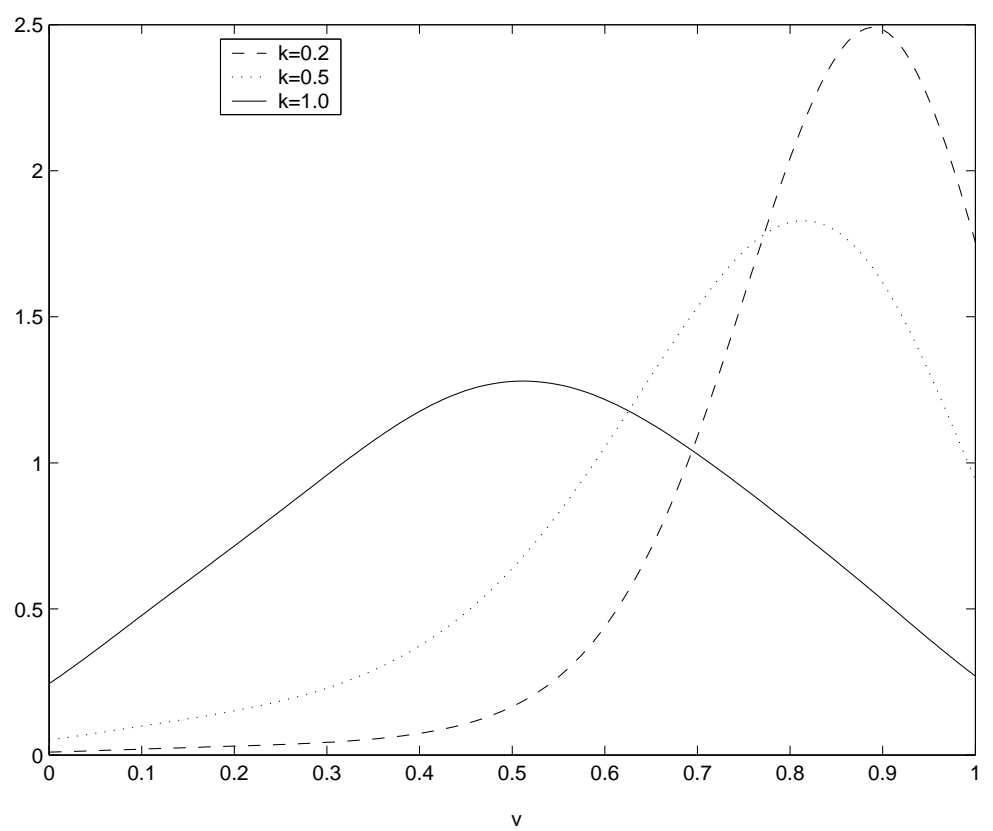

Figure 1. Equilibrium solutions to the Illner, Klar et al. model for varying $k$

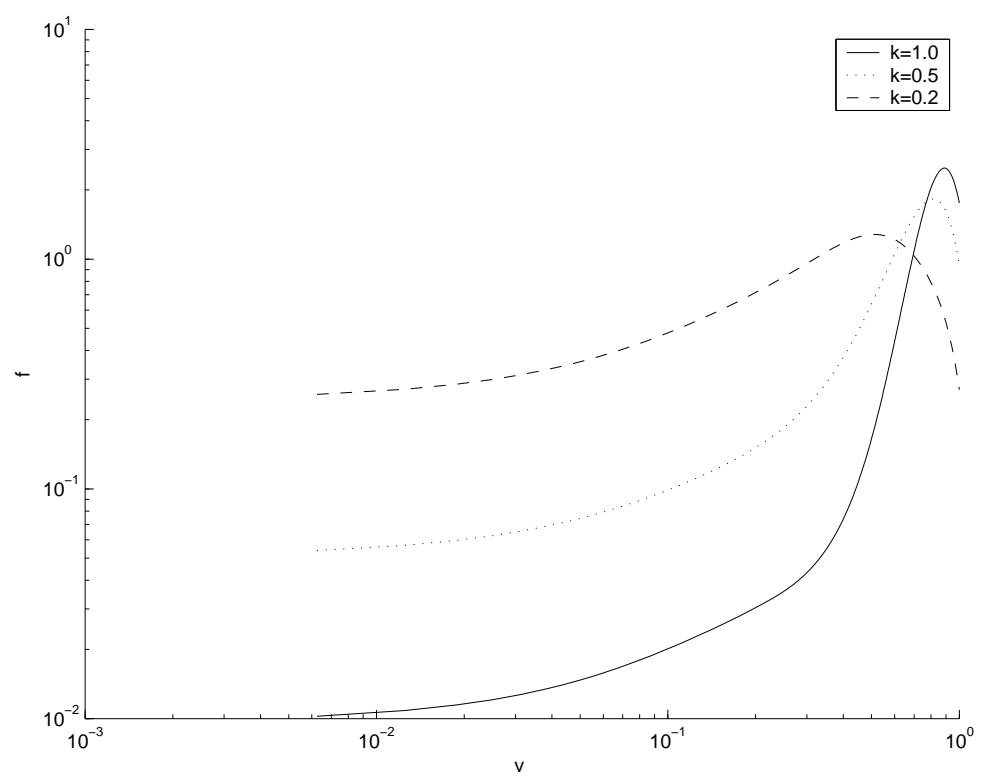

Figure 2. Equilibrium solutions to the Illner, Klar et al. model for varying $k$ in the log-log plot 


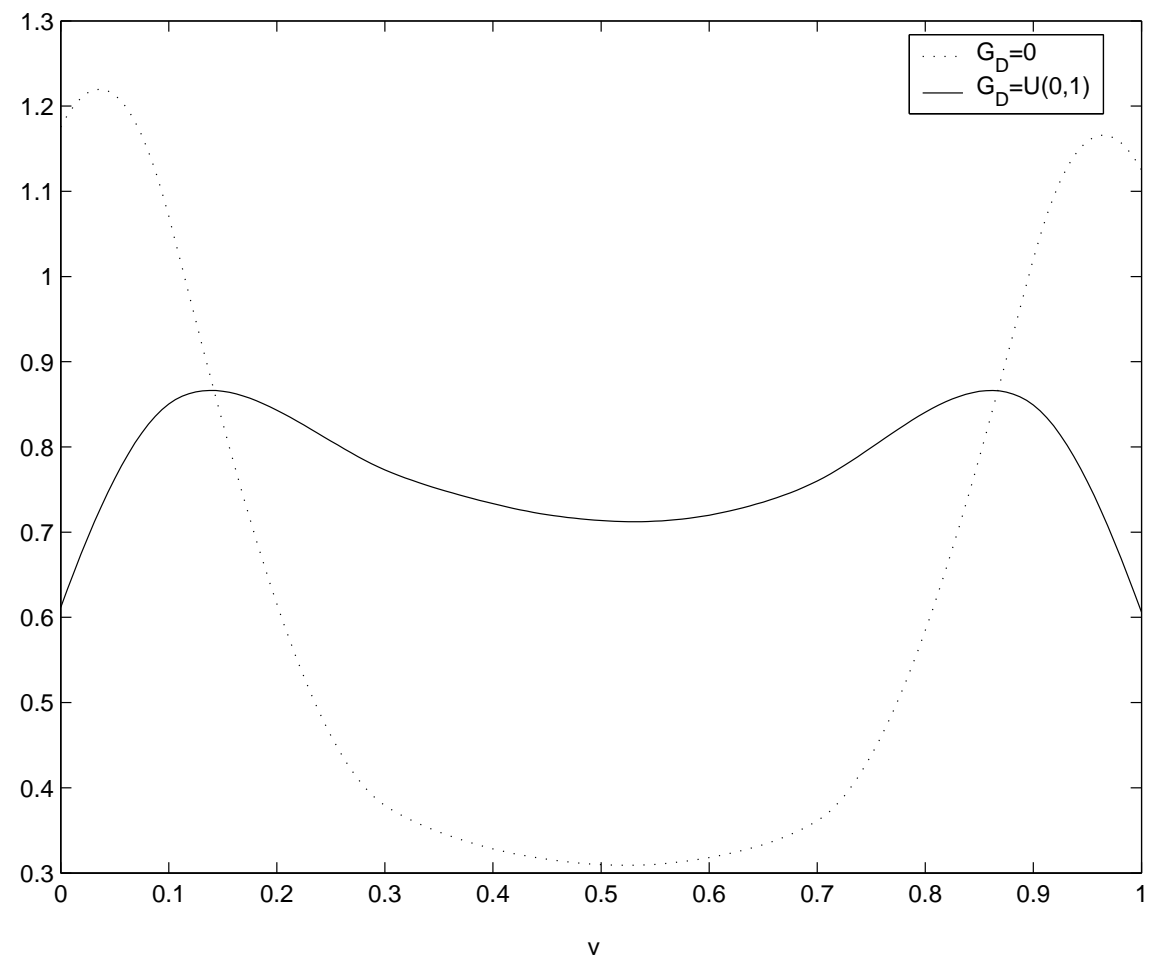

Figure 3. Equilibrium solutions to the simplified model of Klar, et al. with and without the source term

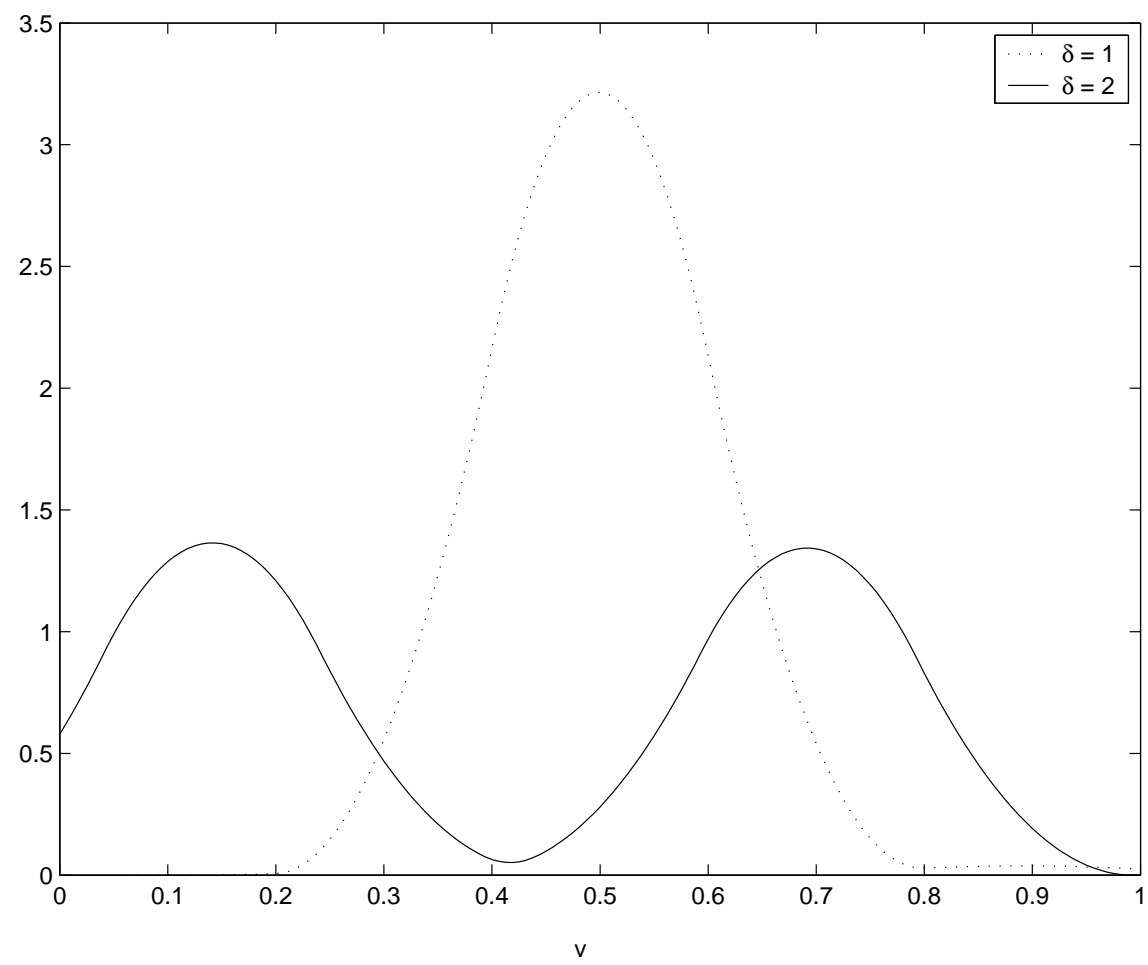

Figure 4. Equilibrium solutions to Helbing-like models for different choices of $\delta$ 


\section{Conclusions}

- A Monte-Carlo code for homogeneous kinetic traffic flow equations has been developed. A large class of different interaction laws can be treated numerically in a uniform way.

- Various existing kinetic traffic flow models have been reformulated and included in the general setting to allow the application of the new Monte-Carlo algorithm.

- Further numerical comparisons with discrete ordinate methods, i.e., deterministic schemes for the traffic flow models will be investigated in a forthcoming paper.

- Inhomogeneous situations can be treated as well using the Monte-Carlo methods, as, for example, for the Enskog equation in rarefied gas dynamics. Further numerical studies and comparison of computation times to the already existing deterministic codes will be considered in a future work.

\section{Acknowledgements}

Part of the present work was supported by Deutsche Forschungsgemeinschaft (DFG), KL 1105/5 and by the European Union financed network no. HPRN-CT-2002-00282. M. Herty would like to thank the Department for Mathematics in Ferrara for their hospitality.

\section{References}

[1] G. Bird, Molecular gas dynamics, Clarendon Press, Oxford, 1976.

[2] M. Günther, A. Klar, T. Materne, and R. Wegener, An explicitly solvable kinetic model for vehicular traffic and associated macroscopic equations, Math. Comp. Modelling, 35 (2002), p. 591.

[3] D. Helbing, Traffic and related self-driven many-particle systems, Reviews of Modern Physics, 73 (2001), pp. $1067-1141$.

[4] R. Illner, A. Klar, H. Lange, A. Unterreiter, and R. Wegener, A kinetic model for vehicular traffic: Existence of stationary solutions, J. Math. Anal. Appl., (1999).

[5] A. Klar and R. Wegener, Enskog-like kinetic models for vehicular traffic, J. Stat. Phys., 87 (1997), pp. 91-114.

[6] A. Klar and R. Wegener, A hierachy of models for multilane vehicular traffic I: Modeling, SIAM J. Appl. Math., 59 (1998), pp. 983-1001.

[7] A. Klar and R. Wegener, A hierachy of models for multilane vehicular traffic II: Numerical investigations, SIAM J. Appl. Math., 59 (1998), pp. 1002-1011.

[8] A. Klar and R. Wegener, Kinetic derivation of macroscopic anticipation models for vehicular traffic, SIAM J. Appl. Math., 60 (2000), pp. 1749-1766.

[9] K. Nanbu, Direct simulation scheme derived from the Boltzmann equation, J.Phys. Soc. Japan, 49 (1980), pp. 2042-2049.

[10] L. Pareschi and G. Russo, An introduction to Monte Carlo methods for the Boltzmann equation, in: ESAIM: Proceedings, vol. 10, 2001, p. 35.

[11] L. Pareschi and G. Russo, Time Relaxed Monte Carlo methods for the Boltzmann equation, SIAM J. Sci. Comp., 23 (2001), p. 1253.

[12] S. Paveri-Fontana, On Boltzmann like treatments for traffic flow, Transportation Research, 9 (1975), pp. 225-235. 
[13] W. Phillips, Kinetic Model for Traffic Flow, National Technical Information Service, Springfield, Virginia, 1977.

[14] I. Prigogine and R. Herman, Kinetic Theory of Vehicular Traffic, American Elsevier Publishing Co., New York, 1971.

[15] M. Schreckenberg, A. Schadschneider, K. Nagel, and N. Ito, Discrete stochastic models for traffic flows, Physical Review E, 51 (1995), p. 2939. 